\title{
Application of a Bayesian Spatiotemporal Surveillance Method to NYC Syndromic Data
}

\author{
Alison Levin-Rector*1, Ana Corberán-Vallet ${ }^{3}$, Andrew B. Lawson ${ }^{2}$, Ramona Lall ${ }^{1}$ and Robert \\ Mathes ${ }^{1}$
}

${ }^{1}$ NYC Dept of Health and Mental Hygiene, Queens, NY, USA; ${ }^{2}$ Medical University of South Carolina, Charleston, SC, USA; ${ }^{3}$ University of Valencia, Valencia, Spain

\section{Objective}

To adapt a previously described Bayesian model-based surveillance technique for cluster detection [1] to NYC Emergency Department (ED) visits.

\section{Introduction}

As technology advances, the implementation of statistically and computationally intensive methods to detect unusual clusters of illness becomes increasingly feasible at the state and local level [2]. Bayesian methods allow for the incorporation of prior knowledge directly into the model, which could potentially improve estimation of expected counts and enhance outbreak detection. This method is one of eight being formally evaluated as part of a grant from the Alfred P. Sloan Foundation.

\section{Methods}

We mimicked prospective surveillance by applying a Bayesian model to daily respiratory syndrome counts across 180 residential zip codes in NYC ED data in 2009, the year of the H1N1 influenza pandemic [3]. We chose a 15-day moving baseline period against which we compared the subsequent day's data to check for unusual levels of respiratory visits.

Inputs to the model included the spatial distribution of zip codes within NYC, day-of-week and holiday indicators, and background population effects. Population effects were calculated based on average daily visits in the baseline period and zip code-level population according to the 2010 census. The inputs were combined in a hierarchical model to estimate zip code-specific expected respiratory visits, and alarms were sounded on days for which observed values of respiratory visits within a zip code diverged from expected values as measured by a Bayesian diagnostic.

To determine an appropriate critical level, above which an alarm is sounded, we simulated outbreak-free respiratory counts and ran the method for each day in a 2-year artificial time series [4]. The critical level was chosen to be that which limited alarms to one every 100 days on average across all zip codes.

\section{Results}

Citywide, 56 alarms were produced across 15 zip codes in 2009. All occurred between mid-May and mid-June, and the first alarm sounded in northeast Queens. These findings correspond to the course of the H1N1 pandemic in NYC [5]. We illustrate resulting alarms for a sample zip code (Figure). In this zip code, 12 alarms sounded within a 13 day period in late May, and all occurred during days of elevated respiratory visits.

\section{Conclusions}

The application of this Bayesian model-based surveillance technique on respiratory ED visits in NYC in 2009 produced promising preliminary results. Alarms occurred during high levels of respiratory visits, but given the magnitude of the H1N1 pandemic, we would have expected to see alarms across a higher number of zip codes. This highlights the first major challenge: setting thresholds. More investigation is needed to determine an appropriate critical level.

Another potential challenge in implementing this method is choosing an appropriate length of baseline. We chose a 15-day baseline in part because of computational limitations. As the length of the baseline increases, runtime and memory constraints become a barrier.

Future work includes evaluating our choice of baseline length, considering other alarm thresholds, and conducting a formal evaluation of the method across five syndromes in NYC.

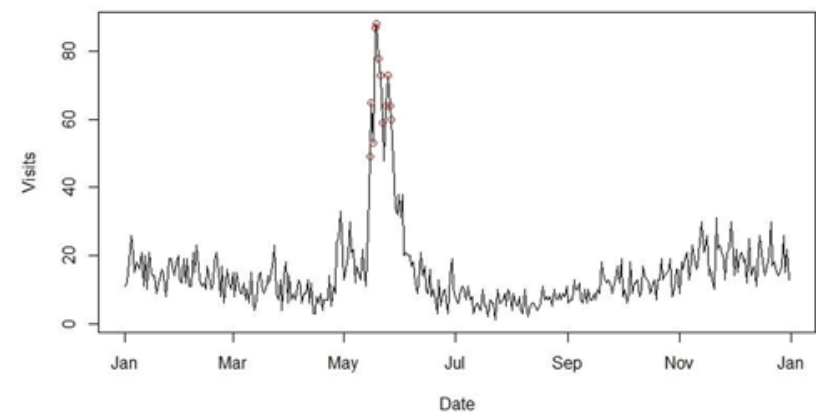

Daily ED visits for respiratory syndrome and alarms in a sample zip code, 2009

\section{Keywords}

syndromic surveillance; Bayesian models; outbreak detection; spatiotemporal data

\section{References}

1. Corberan-Vallet A, Lawson AB. Conditional predictive inference for online surveillance of spatial disease incidence. Statistics in medicine. 2011;30(26):3095-116.

2. Unkel S, Farrington CP, Garthwaite PH. Statistical methods for the prospective detection of infectious disease outbreaks: a review. J R Statist Soc A. 2012;175(1):49-82.

3. Centers for Disease Control and Prevention. The 2009 H1N1 Pandemic: Summary Highlights, April 2009-April 2010 [cited 2013 August 22]. Available from: http://www.cdc.gov/h1n1 flu/cdcresponse.htm.

4. Lotzel T, Shmueli G, Yahav I. Simulating Multivariate Syndromic Time Series and Outbreak Signatures. Smith School of Business: University of Maryland, 2007.

5. NYC Department of Health and Mental Hygiene. Pandemic Influenza Surveillance in New York City, 2009-10. Epi Data Brief. 2011;5.

\footnotetext{
*Alison Levin-Rector

E-mail: alevinrector@health.nyc.gov
} 